\title{
Sistemas produtivos de caprinocultura leiteira no semiárido nordestino: controle integrado das parasitoses gastrointestinais visando contornar a resistência anti-helmíntica ${ }^{1}$
}

\author{
Beatriz Riet-Correa $^{2 *}$, Sara Vilar Dantas Simões ${ }^{3}$ e Franklin Riet-Correa ${ }^{3}$
}

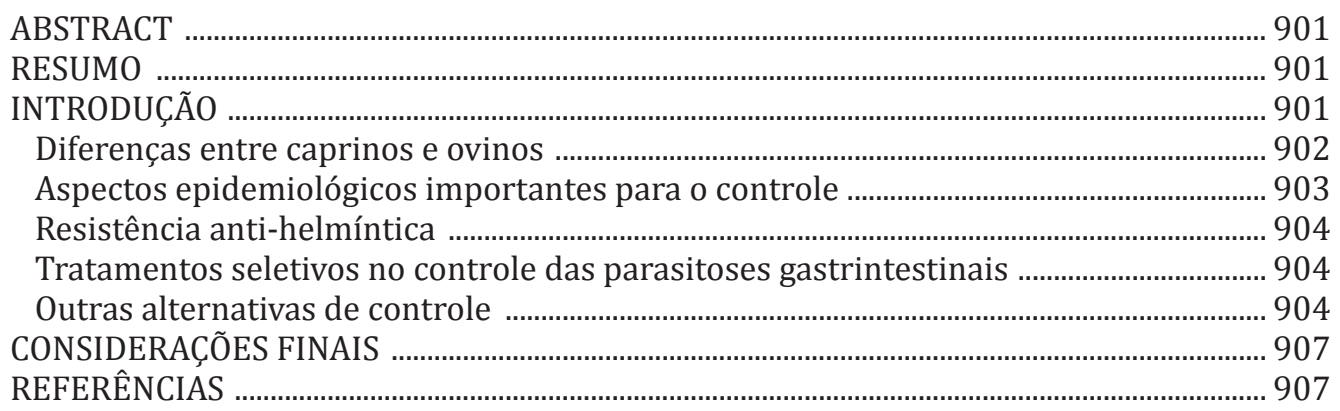

\begin{abstract}
Riet-Correa B., Simões S.V.D. \& Riet-Correa F. 2013. [Dairy goat production in the Brazilian semiarid region: integrated gastrointestinal nematodes control to overcome anti-helminthic resistance.] Sistemas produtivos de caprinocultura leiteira no semiárido paraibano: controle integrado das parasitoses gastrointestinais visando contornar a resistência anti-helmíntica. Pesquisa Veterinária Brasileira 33(7):901-908. Hospital Veterinário, Universidade Federal de Campina Grande, Av. Universitária s/n, Patos, PB 58700-970, Brazil. E-mail: beatrizriet@hotmail.com

Goats are very important for the economy of the semiarid region of northeastern Brazil; however, the high frequency of parasitism by gastrintestinal nematodes (GIN) and the increase of anthelmintic resistance are threatening goat industry in the region. In this paper we review the control strategies for GIN in goats including: 1) the differences between goats and sheep to GIN infections; 2) important epidemiological aspects to consider in the control; and 3) technologies to be used for integrated control of GIN and anti-helmintic resistance.
\end{abstract}

INDEX TERMS: Anti-helminthic resistance, gastrointestinal nematodes, goats, integrated control, semiarid.

RESUMO.- A caprinocultura é muito importante para a economia do semiárido nordestino; no entanto a alta frequência das parasitoses gastrintestinais e o aumento da resistência parasitária ameaçam gravemente essa atividade. Nesta revisão são discutidos vários aspectos importantes para o controle das parasitoses gastrintestinais de caprinos, incluindo: 1) as diferenças entre caprinos e ovinos;

\footnotetext{
${ }^{1}$ Recebido em 4 de março de 2013.

Aceito para publicação em 19 de junho de 2013.

${ }^{2}$ Programa de Pós-Graduação em Medicina Veterinária, Universidade Federal de Campina Grande (FCG), Hospital Veterinário, Avenida Universitária s/n, Patos, PB 58700-970, Brasil. *Autor para correspondência: beatrizriet@hotmail.com

${ }^{3}$ Hospital Veterinário, UFCG, Avenida Universitária s/n, Patos, PB.
}

2) aspectos epidemiológicos importantes a serem levados em consideração para o controle; e 3) tecnologias a serem utilizadas para realizar o controle das parasitoses gastrintestinais em forma integrada e diminuir a frequência de resistência anti-helmíntica.

TERMOS DE INDEXAÇÃO: Caprinos, controle integrado, parasitoses gastrintestinais, resistência anti-helmíntica, semiárido.

\section{INTRODUÇÃo}

Doenças causadas por nematóides gastrointestinais (NGI) são um dos fatores que mais afetam a produtividade de pequenos ruminantes (Hoste et al. 2005, Costa et al. 2009), causando muitas perdas econômicas não somente por morte de animais, mas também por queda na produção (dimi- 
nuição do ganho de peso e da produção de lã e leite) (Hart 2011, Torres-Acosta et al. 2012). Parasitas gastrointestinais são normais, porém, o excesso de parasitas é patogênico. É necessário manter uma baixa infestação de parasitas, para manter o sistema imunológico sempre ativo para combater a infecção, porém, devem ser adotadas várias estratégias que, combinadas, evitem que o número de parasitas atinja um nível patogênico (Hart 2011, Torres-Acosta et al. 2012). O NGI mais patogênico para ovinos e caprinos, principalmente no nordeste, é Haemoncus contortus, responsável por grandes perdas produtivas nessas espécies (Costa et al. 2011). No semiárido, além de $H$. contortus ser o parasita mais frequente é o único descrito como responsável pela ocorrência de surtos de parasitoses gastrintestinais (Costa et al. 2009); portanto o controle de parasitas gastrintestinais no semiárido deve ser baseado, principalmente, no controle de $H$. contortus.

Durante décadas os anti-helmínticos (ANTH) foram utilizados eficientemente no controle dos NGI, entretanto, a consequência natural do uso indiscriminado desses químicos é o desenvolvimento da resistência anti-helmíntica (RA) (Hoste et al. 2005, Hart 2011), definida como sendo a capacidade de uma população de parasitas sobreviver a doses de ANTH que poderiam ser letais para populações susceptíveis (Torres-Acosta \& Hoste 2008). A RA tem se tornado um problema mundial na criação de pequenos ruminantes, e tem sido relatada em diversos países (Chartier et al. 1998, Van Wyk et al. 1999, Pomroy 2006), incluindo o Brasil (Santos \& Gonçalves 1967, Echevarria \& Trindade 1989, Molento et al. 2011). Na região Nordeste do Brasil já foi relatada em caprinos em Pernambuco (Charles et al. 1989), Bahia (Barreto \& Silva 1999), Ceará (Vieira \& Cavalcante 1999, Melo et al. 2003), Rio Grande do Norte (Coelho 2009) e Paraíba (Lima et al. 2010, Riet-Correa 2013). Possivelmente a principal causa do desenvolvimento de RA no Nordeste é o tratamento anti-helmíntico repetido de caprinos e ovinos durante a seca (Costa et al. 2011). Além disso, em caprinos a utilização de doses semelhantes às dos ovinos tem levado sistematicamente ao tratamento com subdoses o que também pode ter favorecido o aparecimento de resistência anti-helmíntica (Costa et al. 2011).

Em um trabalho recente foi encontrada resistência múltipla a todos os ANTH testados (lactonas macrocíclicas, salicilanilidas, benzimidazóis, fosforados (triclorfom) e imidotiazóis) em sete fazendas produtoras de leite de cabra no semiárido Paraibano (Riet-Correa 2013, Riet-Correa et al. 2013). Devido à importância da caprinocultura na região Nordeste e ao aumento da RA, é necessário revermos as estratégias de controle das parasitoses adotadas até o momento e mudarmos urgentemente nossos conceitos, a fim de diminuir o avanço da RA antes que a criação de caprinos na região seja inviabilizada. Nesta revisão são discutidos vários aspectos importantes para o controle das parasitoses gastrintestinais de caprinos, incluindo: 1) as diferenças entre caprinos e ovinos; 2) aspectos epidemiológicos importantes a serem levados em consideração para o controle e, 3) tecnologias a serem utilizadas para realizar o controle das parasitoses gastrintestinais em forma integrada e diminuir a frequência de resistência anti-helmíntica.

\section{Diferenças entre caprinos e ovinos}

Caprinos e ovinos são infectados pelas mesmas espécies de NGI, que provocam alterações patológicas e consequências econômicas similares. Apesar do número de caprinos e ovinos no mundo ser similar, a maioria dos estudos a respeito da interação hospedeiros-nematóides e controle de espécies de NGI tem sido realizados em ovinos. Por muitos anos, os resultados obtidos de pesquisas realizadas com ovinos foram aplicados da mesma forma para caprinos. Entretanto, existem grandes diferenças na resposta contra a infecção entre as duas espécies:

Hábitos alimentares e resistência. Caprinos e ovinos desenvolveram diferentes hábitos alimentares. Ovinos preferentemente pastejam (se alimentam de pastagens ou do extrato herbáceo) e caprinos preferem o ramoneio (hábito de ingerir ramos e folhas de plantas arbustivas). Devido às pastagens serem uma fonte de contaminação por estágios infectantes (L3) de NGI, os ovinos se infectam mais facilmente durante o pastoreio e, por terem evoluído nestas condições, em maior contato com os parasitas, desenvolveram maior imunidade. Os caprinos, pelo contrário, se desenvolveram evitando os parasitas gastrintestinais pelo hábito do ramoneio, portanto com menor contato com os mesmos do que os ovinos, o que levou a uma maior susceptibilidade evidenciada pela menor capacidade de criar uma resposta imune (Hoste et al. 2010). A consequência mais evidente dessa diferença evolutiva é que em rebanhos mistos, em pastagens, os caprinos são mais afetados do que os ovinos (Costa et al. 2011). Além disso, os caprinos demoram mais para desenvolver uma resposta imune (aprox. 12 meses) e não apresentam grandes diferenças na susceptibilidade entre animais jovens e adultos. Pelo contrário, em ovinos, que desenvolvem resposta imune mais rápida (6 meses aproximadamente) e mais eficiente, há diferenças importantes na susceptibilidade entre animais jovens (mais susceptíveis) e animais adultos (mais resistentes) (Hoste et al. 2010).

Metabolização de anti-helmínticos. Cabras toleram melhor as toxinas naturais, em particular metabólitos secundários de plantas (PSMs). Essa característica deve-se, provavelmente, a alimentação dos caprinos (ramoneio) com plantas arbustivas com maior conteúdo em PSMs, que resultou em um processo evolutivo com o desenvolvimento de mecanismos fisiológicos e metabólicos adaptativos para tolerar a toxicidade dos PSMs. Alguns estudos tem demonstrado que essa adaptação metabólica para PSMs naturais acaba tendo consequências farmacológicas e farmacocinéticas a outros xenobióticos, incluindo drogas terapêuticas. Por isso, os caprinos metabolizam os ANTH mais rapidamente do que os ovinos e, portanto, devem ser tratados com doses maiores (Torres-Acosta \& Hoste 2008). Entretanto, até hoje, no Brasil, não existe discriminação nas bulas de medicamentos entre as doses para essas duas espécies. Esse fator levou a uma maior disseminação de RA na população caprina (Costa et al. 2011). As doses de ANTH, ajustadas as espécies, são apresentadas Quadro 1.

Automedicação. Tem sido constatado, também, que em consequência do processo evolutivo mencionado anteriormente, as cabras desenvolveram o hábito de se "auto- 
Quadro 1. Anti-helmínticos comerciais de largo espectro usados em pequenos ruminantes,doses para caprinos e ovinos e vias de administração

\begin{tabular}{lll}
\hline Princípio ativo & Dose $(\mathrm{mg} / \mathrm{kg})$ & Vias de adm. \\
\cline { 2 - 2 } & Ovinos Caprinos & \\
\hline
\end{tabular}

\begin{tabular}{lccc}
\hline Benzimidazois & 50 & 100 & O* \\
Thiabendazole & 5 & 10 & O, IR \\
Albendazole & 15 & 30 & O \\
Mebendazole & 5 & 10 & O, IR \\
Fenbendazole & 5 & 10 & O, IR \\
Oxfendazole & 5 & 10 & O, IR \\
Febantel & 7,5 & 15 & O \\
Netobimin & 7,5 & 12 & O, SC \\
Imidazotiazois/Tetrahidropirimidinas & & 0 \\
Levamisol & 6 & 10 & \\
Morantel & & & $0, \mathrm{SC}, \mathrm{T}$ \\
Lactonas Macrocíclicas & 0,2 & 0,3 & $\mathrm{SC}$ \\
Ivermectina & 0,2 & 0,2 & $\mathrm{O}, \mathrm{SC}$ \\
Doramectina & 0,2 & 0,2 & $\mathrm{~T}$ \\
Moxidectina & 0,5 & 1,0 & \\
Eprinomectina &
\end{tabular}

${ }^{*} \mathrm{O}=$ oral, IR= intra-ruminal, $\mathrm{SC}=$ subcutâneo, $\mathrm{T}=$ tópico. Fonte: Torres-Acosta \& Hoste (2008).

-medicar", isto é, escolher para sua alimentação plantas com efeito anti-helmíntico, que contêm PSMs (Hoste et al. 2010, Torres- Acosta et al. 2012). Este comportamento já foi observado, também, em cordeiros parasitados, que mostraram preferência por plantas que contém taninos e tem efeito anti-helmíntico (Villalba et al. 2010) .

\section{Aspectos epidemiológicos importantes para o controle}

Idade. Como mencionado anteriormente, em caprinos os níveis de infecção são semelhantes entre em jovens e adultos (Hoste et al. 2010); no entanto, fêmeas primíparas apresentam um maior nível de infestação por NGI do que fêmeas multíparas. Essa susceptibilidade foi observada tanto em ovelhas (Hoste el al. 2006) quanto em cabras (Hoste et al. 2001, 2002b). Na Paraíba, em surtos de hemoncose, a maior mortalidade dentro do rebanho ocorreu em cabras nas primeiras semanas após o parto (dados não publicados).

Produção. Vários estudos realizados com caprinos demonstraram que a produção de leite está associada com o nível de infecção por nematóides gastrointestinais. Cabras que tiveram maior produção leiteira foram as mais susceptíveis e mais infestadas por NGI do que as cabras com menor produção de leite (Chartier \& Hoste 1997, Hoste et al. 2002b). Essa mesma suscetibilidade não foi observada em ovinos, provavelmente devido a produção de leite nessa espécie ser aproximadamente duas vezes menor do que nas cabras. É provável que essa diferença seja devida à menor demanda nutricional em ovelhas lactantes do que em cabras lactantes (Hoste et al. 2006).

Aumento da eliminação de ovos no periparto. Este fenômeno tem grande importância epidemiológica em caprinos, não só pelo fato de haver um aumento na eliminação de ovos nesse período, resultando numa maior contaminação ambiental (Torres- Acosta \& Hoste 2008, Costa et al. 2011), mas também por que as cabras são altamente susceptíveis as parasitoses gastrintestinais nessa fase.
Lotação. Estudos em diferentes ambientes tem confirmado a existência de uma relação entre o nível de infecção do hospedeiro e a taxa de lotação. Em criações extensivas, em áreas de caatinga, com um ou menos de um caprino por hectare, não é necessário tratar o rebanho para controlar as parasitoses gastrintestinais. Com a evolução da caprinocultura no semiárido foram também aumentando as taxas de lotação, o que levou a uma alta frequência de surtos de parasitoses gastrintestinais em caprinos. A redução da taxa de lotação tem sido uma medida efetiva para limitar a carga parasitária em ovinos e caprinos (Torres-Acosta \& Hoste 2008). Há informações de que com até cinco ovinos ou cabras por hectare a lotação não tem muita influência na frequência das parasitoses gastrintestinais, mas os riscos aumentam a partir dessa lotação (Hart 2011). Entretanto, existem fatores que interferem na dinâmica da infecção, como: os gêneros dos parasitas, as espécies da pastagem e as condições climáticas. Por essa razão, é difícil indicar um limite geral de taxa de lotação em que a infecção é minimizada (Torres-Acoste \& Hoste 2008).

Confinamento. 0 confinamento é desfavorável para infecções por NGI, isso se deve ao fato que a forma larval infectante (L3) não sobrevive no feno ou silagem. Os tratamentos com ANTH nesse sistema de produção são geralmente desnecessários. (Torres-Acosta \& Hoste 2008). No entanto é necessário levar em conta as condições de higiene das instalações e manejo de forragem, pois é possível o desenvolvimento de larvas infectantes em restos de forragem que permaneçam no solo ou nos coxos por períodos suficientes para o desenvolvimento das mesmas. Por outro lado, quando se utiliza forragem verde é possível que este provenha de pastagens infectadas.

Resistência. Existem diferenças genéticas na susceptibilidade a NGI em ovinos e caprinos, que podem ser entre raças (algumas raças são mais resistentes) e dentro da mesma raça (Hart 2011). Em ovinos, foi demonstrado que a raça Santa Inês é mais resistente a NGI que a Sulfock ou Ille de France (Amarante et al. 2004). Na Paraíba, em condições semelhantes, os caprinos Boer e os ovinos Dorper demonstraram serem mais susceptíveis do que as raças nativas (Costa et al. 2011). 0 uso da seleção genética em ruminantes para características de resistência a infecção por NGI tem sido apresentado como uma ferramenta no controle sustentável de parasitas. Estudos demonstram que a seleção genética, após várias gerações, reduzem substancialmente a excreção fecal de ovos e a contaminação da pastagem (Hoste \& Torres-Acosta 2011). Portanto, é importante que sejam selecionados os animais mais resistentes dentro do rebanho, procedendo com o descarte dos animais susceptíveis.

Região. Devido ao parasitismo por NGI estar distribuído mundialmente, é impossível descrever a dinâmica da infecção nas diferentes latitudes (Torres- Acosta \& Hoste 2008). O clima é um fator importante na dinâmica da infecção, pois é o que vai determinar o tempo de sobrevivência da forma infectante (L3) na pastagem. Em climas tropicais e subtropicais a sobrevivência da L3 é relativamente curta, de um a três meses (Torres-Acosta \& Hoste 2008) e há autores que mencionam um período de 40 dias para descon- 
taminar a pastagem (Hart 2011). Já em climas temperados as larvas podem sobreviver por até 18 meses. Entretanto, a L3 é sensível a situações de seca prolongada, assim como a longos períodos de frio (O'Connor et al. 2006, Hoste \& Torres- Acosta 2011).

Sistema de produção. Como mencionado anteriormente, a frequência das PGI depende em grande parte do sistema de produção. Isso significa que, principalmente em pastagens, o sistema mais produtivo, com animais de alta produtividade, pode não ser economicamente mais rentável, pois podem ser necessários investimentos maiores para o controle das PGI e, a longo prazo, a resistência anti-helmíntica pode impossibilitar a produção. Portanto maiores produtividades exigem melhores técnicas de controle de NGI, e alternativamente, o produtor pode optar por ter animais menos produtivos, porem mais resistentes às parasitoses gastrintestinais e com menores gastos para o controle das mesmas (Hart 2011).

\section{Resistência anti-helmíntica}

Atualmente qualquer proposta de controle das parasitoses gastrintestinais deve ter dois objetivos: evitar a ocorrência da doença e evitar o aparecimento ou o aumento da resistência anti-helmíntica. Para isso devemos conhecer os fatores que favorecem a resistência e os fatores que evitam ou retardam o seu aparecimento.

Fatores favoráveis à resistência anti-helmíntica. Os fatores que favorecem o desenvolvimento de resistência são aqueles que procuram eliminar todos os parasitas susceptíveis da população de caprinos, dando possibilidades de sobrevivência aos resistentes, principalmente quando não há parasitas na refugia (população de NGI que estão na pastagem e não são expostos ao tratamento). Dentre estes fatores temos: tratar todo o rebanho simultaneamente; tratar o rebanho e mudar o mesmo para uma área livre de parasitas; tratamentos frequentes ou supressivos, tentando diminuir ao máximo as parasitoses gastrintestinais; tratamentos sistemáticos (periódicos) de todo o rebanho; tratamento inadvertido (utilização de ANTH para tratamento de ectoparasitas, por exemplo); tratamento durante a seca; tratamento com subdoses (caprinos com doses de ovinos por exemplo); utilização do mesmo anti-helmíntico por mais de um ano; comprar animais com helmintos resistentes e introduzi-los no rebanho sem tratamento prévio eficiente; utilizar unicamente drogas, sem considerar outras alternativas (Chartier \& Hoste 1997, Torres- Acosta \& Hoste 2008, Jackson et al. 2012).

Fatores que previnem à resistência anti-helmíntica. As medidas que podem ser utilizadas para prevenir a RA são: tratar os animais após a mudança de campo para evitar a infecção da pastagem apenas com parasitas resistentes; tratar caprinos com doses corretas; mudar de anti-helmíntico anualmente; comprovar, mediante OPG, a eficiência do tratamento; realizar teste de resistência para mudar de anti-helmíntico; diminuir o número de tratamento utilizando outras alternativas de controle; tratar durante a chuva, quando as pastagens estão infectadas; tratamentos seletivos ( Torres- Acosta \& Hoste 2008, Hart 2011).

\section{Tratamentos seletivos no controle das parasitoses gas- trintestinais}

Uma das melhores ferramentas ao alcance dos produtores são os tratamentos seletivos, onde é tratada somente uma parte do rebanho. Tem sido demonstrado que apenas 20\%-30\% dos animais carregam 70\%-80\% dos NGI adultos em um rebanho (Rinaldi \& Cringoli 2012); portanto, se conseguirmos identificar esses animais dentro de um rebanho e administrar ANTH somente para essa população, aumenta-se a refugia, diminuindo o risco de RA (Hart 2011). 0 tratamento seletivo mais utilizado é o FAMACHA, que considera que ovinos ou caprinos infectados por Haemonchus contortus, o parasita hematófago de maior importância em pequenos ruminantes, podem ser facilmente identificados observando a coloração da mucosa ocular dos animais utilizando um cartão a fim de identificar o grau de anemia e os animais que necessitam tratamento. O FAMACHA, em geral, tem dado resultados positivos, diminuindo o número de dosificações, contornando o problema da resistência e ajudando a identificar aos animais mais susceptíveis (Van Wyk \& Bath 2002, Molento 2008, Molento et al. 2011). No entanto, em um trabalho recente, a utilização do FAMACHA durante a seca resultou em maior número de tratamentos. A carência alimentar, normalmente observada nos períodos secos da região em estudo, provavelmente, dificultou a recuperação dos animais com anemia e levou estes a serem dosificados diversas vezes, mesmo com infecções baixas (Riet-Correa 2013).

0 tratamento seletivo pode ser realizado com base em outros parâmetros incluindo idade ou estado fisiológico, o escore corporal, a presença de algum sinal clínico (condição do pelame, edema submandibular, diarreia, e descarga nasal (Torres- Acosta et al. 2012). As categorias mais susceptíveis a serem escolhidas para realização do tratamento seletivo são as fêmeas no periparto, principalmente as primíparas e as cabras multíparas de alta produção leiteira (Chartier \& Hoste 1997, Hoste et al. 2002a). Também pode ser realizado o tratamento de uma parte do rebanho, deixando os animais em melhor estado corporal ou das categorias mais resistentes (cabras solteira adultas, por exemplo) sem tratar. 0 tratamento na presença de sinais clínicos, assim como o FAMACHA, em regiões onde $H$. contortus é o principal parasita tem o problema que os animais podem passar um tempo demasiadamente prolongado para se recuperar, e, em casos de infecções agudas podem morrer. Neste caso, o exame de fezes com contagem de ovos apesar de mais caro, pareceria ser mais adequado, por que os animais podem ser tratados antes de apresentar sinais clínicos do parasitismo.

\section{Outras alternativas de controle}

Pastejo rotativo. 0 objetivo geral de métodos de manejo na pastagem é limitar o contato entre os hospedeiros susceptíveis e os estágios infectantes do parasita (Hoste \& Torres- Acosta 2011). É praticamente impossível utilizar pastagens irrigadas com pequenos ruminantes sem um pastejo rotativo que controle a infecção por NGI. Para um manejo adequado da pastagem, é necessário o conhecimento da epidemiologia da região. Na pastagem, os ovos 
dos PGI evoluem para L3 (3o estágio larval, responsável pela infecção no hospedeiro) em 4-7 dias. Portanto, em um pastejo rotativo, para evitar as reinfecções os animais não deveriam permanecer mais do que quatro dias na mesma pastagem. A taxa de sobrevivência da L3, que determina o período de rotação em que as pastagens devem ficar sem animais, depende das condições climáticas. Nas áreas subtropicais e tropicais L3 se mantem no ambiente por um a três meses. Já em climas temperados, estas podem sobreviver de seis a 18 meses (Torres-Acosta \& Hoste 2008).

Pomroy et al. (2003) obteve bons resultados na diminuição da contaminação da pastagem utilizando um pastejo rotacionado de curta duração, com um longo período de descanso. Ele dividiu uma área em 14 piquetes, onde os animais permaneciam em cada piquete por cinco dias, totalizando no final, 65 dias de descanso da pastagem. No entanto, um longo período de rotação pode diminuir significativamente a qualidade da pastagem. Por esta razão o período de rotação não deveria ser maior do que 35 dias. Um sistema de rotação para caprinos, no semiárido, pode ser realizado com 12 parcelas, com permanência durante três dias nas parcelas e um período total de rotação de 36 dias. Barger et al. (1994), nas Ilhas Fiji, utilizaram o pastejo rotativo com 10 piquetes e um período de permanência de 3,5 dias, para que as mudanças de piquetes ocorressem sempre no mesmo dia da semana. Outra alternativa é utilizar junto com os caprinos um rebanho de outra espécie: ovinos adultos, que são mais resistentes do que os caprinos apesar de se infectarem com os mesmos parasitas; ou bovinos ou equinos que não se infectam com os mesmos parasitas (Barger 1997). Neste caso podemos utilizar, por exemplo, uma rotação com 22 piquetes com três dias de pastejo. Enquanto os caprinos iniciam o pastejo no piquete 1 o outro rebanho inicia no piquete 11. Quando os caprinos cheguem ao piquete 11 o outro rebanho estará no piquete 1 e as pastagens terão um período de descanso de 30 dias.

Outras medidas de manejo de pastagens. Mesmo sem utilizar o pastejo rotativo outras medidas de manejo de pastagens contribuem para reduzir a infecção por NGI como o pastejo alternado ou misto de caprinos ou ovinos com outras espécies (bovinos ou equinos). A utilização de diferentes espécies de forrageiras na pastagem utilizando espécies mais rasteiras com espécies mais eretas e plantas arbustivas favorece o controle das NGI, pois monoculturas de plantas que formam uma pastagem fechada de baixa altura, como o tifton (Cynodon dactylon), mantêm um microambiente úmido favorável ao deslocamento da L3 até a altura em que são facilmente ingeridas pelos animais. Pelo contrário, pastagens eretas e altas, como Brachiaria brizantha e Panicum maximum fazem com que os animais pastejem acima da parte da planta em que as L3 se concentram a uma distância de $6-8 \mathrm{~cm}$ do solo (Hart 2011). Por outro lado, o cultivo de espécies arbóreas como leucena (Leucaena leucocephala), gliricidia (Gliricidia sepium), sabiá (Auxema oncocalyx) e maniçoba (Manihot spp.) favorecem o hábito de ramoneio e evitam a ingestão de L3.

Tratamentos corretos com doses corretas. Além dos problemas como sub-dosagens por utilização das mesmas doses para ovinos, outros erros durante o tratamento anti- -helmíntico devem ser evitados. Deve-se ter o cuidado de não administrar produtos vencidos, observar se a pistola está calibrada adequadamente, não dosar animais de pesos diferentes com as mesmas doses e evitar o armazenamento inadequado dos produtos. Todos esses fatores podem levar a realização de tratamentos ineficientes, devendo ser descartadas quando houver a suspeita de RA (Hoste et al. 2010).

Em geral, a resistência anti-helmíntica é irreversível, apesar de que, raras exceções são descritas; Bird et al. (2001) relatam a substituição de helmintos resistentes por helmintos susceptíveis após a utilização de um anti-helmíntico múltiplo (composto por várias drogas).

Tratamento na seca. Para evitar a RA um dos pontos mais importantes é o de evitar os tratamentos durante a seca. A região Nordeste caracteriza-se por duas estações: a seca e a chuva. As recomendações técnicas indicadas até o momento eram de tratar o rebanho quatro vezes por ano, sendo uma no período chuvoso e três no período seco (Embrapa 1994, Sebrae 2010, Codevasf 2011), porém no período seco existem poucas ou nenhuma larva na pastagem, por isso, essa medida pode levar ao rápido aparecimento da RA, pois reduz drasticamente a refugia e somente os genes resistentes na população de parasitas sobrevivem. Portanto, uma medida importante a ser adotada é não tratar todo o rebanho durante a seca. 0 problema é que, ainda durante a seca podem ocorrer infecções por NGI que exigem tratamento (Riet-Correa 2013), portanto, deve-se realizar pelo menos um tratamento nesse período. A melhor solução seria utilizar algum dos tratamentos seletivos mencionados no ponto 4. Provavelmente a solução mais adequada neste caso seja realizar contagem de OPG de todo o rebanho e tratar somente os animais com contagens mais altas, com mais de 500-1000 OPG, por exemplo.

Seleção genética. É possível, mediante a identificação e eliminação dos animais mais susceptíveis do rebanho, aumentar a população de animais resistentes ou resilientes (Hoste et al. 2001). Podem ser utilizadas raças que já se sabe que são mais resistentes á infecção por NGI; no entanto, é essencial que se selecionem os indivíduos mais resistentes dentro de uma mesma raça, pois após algumas gerações selecionando indivíduos resistentes têm-se uma diminuição efetiva na contaminação da pastagem (Hoste \& Torres-Acosta 2011). Em condições de campo, além dos sinais clínicos, o OPG é uma boa ferramenta para selecionar os animais mais resistentes. No entanto, devemos considerar que as cabras que eliminam maior quantidade de ovos são as mais produtivas (Hoste et al. 2002c), pelo que este fator deve, também, ser levado em consideração.

Nutrição. A manipulação nutricional pode ser utilizada como uma ferramenta no controle de infecções por NGI em pequenos ruminantes, reduzindo a dependência de tratamentos convencionais com ANTH (Torres- Acosta et al. 2012). 0 metabolismo proteico é o primeiro afetado quando existe uma infecção por NGI. Por esse motivo, a proteína é o nutriente mais importante para aumentar a resistência/ resiliência do hospedeiro. Porém, quando administrada sem uma fonte de energia, pode não fornecer o efeito esperado nos animais e talvez tenha um efeito adverso por 
excesso de amônia (Hoste et al. 2005, Torres- Acosta et al. 2012). Alguns microelementos, como Se, $\mathrm{Zn}$ e Cu e vitamina E, são importantes para o bom funcionamento do sistema imune. Para isso, é importante, no semiárido, planejar corretamente a nutrição dos caprinos, principalmente durante a seca quando a disponibilidade de forragem é muito baixa. É importante realizar reservas forrageiras e utilizar outro tipo de suplementação, com grãos ou subprodutos. Neste aspecto uma alternativa importante para a região semiárida pode ser a utilização de misturas múltiplas contendo ureia, um concentrado proteico, um concentrado energético, microelementos e, para controlar o consumo, cloreto de sódio (Riet-Correa 2004). A suplementação de fêmeas no periparto alivia parcial ou totalmente o fenômeno do aumento da eliminação de ovos no periparto, quando a demanda por nutrientes é maior, diminuindo significativamente a excreção de ovos nas fezes e o risco de aparecimento de doença clínica. Deve-se suplementar, também, as cabras mais produtivas no pico de lactação (Hoste et al. 2005, Torres-Acosta \& Hoste 2008).

Algumas plantas podem proporcionar, além de nutrientes, um efeito anti-helmíntico direto, que é o caso das plantas ricas em taninos condensados, que aparecem como uma opção para diminuir a carga parasitária dos animais (Alonso-Dias et al. 2010, Muir 2011,Torres-Acosta et al. 2012). Taninos são uma classe de polímeros flavonoides, alguns com efeitos ANTH (Muir 2011) que podem ser encontrados em diversas plantas da caatinga (Araujo Filho \& Carvalho 1998, Monteiro et al. 2005). Algumas pesquisas demonstraram que taninos podem reduzir a excreção de ovos nas fezes em 50\%-80\%. Aparentemente, essa redução é devido a um efeito direto na forma larval juvenil, impedindo seu desenvolvimento. Além desse efeito ANTH direto, essas plantas tem um alto valor proteico, melhorando o sistema imune do hospedeiro (Hart 2011). Portanto, é interessante que haja uma incorporação dessas plantas nos sistemas de pastejo. Uma boa opção para a região Nordeste é a leucena, que além de ter esse efeito ANTH direto, é uma ótima fonte de proteína.

As folhas de mandioca (Manihot esculenta) também demonstraram ter um efeito anti-helmíntico, diminuindo significativamente o OPG em cordeiros (Marie-Magdeleine et al. 2010). Pastagens com outras leguminosas utilizadas no nordeste, mesmo sem efeito anti-helmíntico como a maniçoba, gliricídia, cunhã (Clitoria ternatea), guandú (Cajanus sabia) e feijão bravo (Capparis hastata) são uma fonte importante de proteína, aumentando a resistência contra NGI. Alguns subprodutos como restos de mandioca e folhas de bananeira tem efeito anti-helmíntico e podem ser utilizado como suplemento (Hoste \& Torres-Acosta 2011).

Partículas de óxido de cobre (COWP). Partículas de óxido de cobre (COWP) foram primeiramente desenvolvidas para o tratamento da deficiência de cobre. Porém, pesquisadores na Nova Zelândia relataram que a utilização de COWP em cordeiros reduziu em $90 \%$ o número de parasitas da espécie Haemonchus contortus recuperados na necropsia e $56 \%$ de Ostertargia circumcinta (Bang et al. 1990). A maior limitante para a utilização de COWP em ovinos é o fato de serem muito susceptíveis á intoxicação por cobre.
Caprinos são bem mais resistentes que ovinos e o óxido de cobre incorporado em COWP é menos absorvido que outras formas de cobre, como sulfato de cobre, reduzindo o risco de toxicidade (Torres-Acosta \& Hoste 2008). Em caprinos, COWP nas doses de $0,5 \mathrm{~g}$, consideradas ótimas para reduzir o risco de intoxicação por $\mathrm{Cu}$, foram eficientes em reduzir a eliminação de ovos em cabras jovens e doses de $5 \mathrm{~g}$ foram eficientes em cabras adultas. No entanto, o óxido de Cu não foi eficiente para controlar as infecções recentes de L3 (Burke et al. 2007). Em outro trabalho foi demonstrado que COWP reduz o OPG em cabras, principalmente quando o nematóide predominante é $H$. contortus (Burke et al. 2010). No Brasil, Gonçalves \& Echevarria (2004) obtiveram bons resultados no controle de $H$. contortus utilizando cápsulas gelatinosas com 3,4 gramas de óxido de cobre para ovinos manejados extensivamente. Essa quantidade não causou toxicidade e protegeu contra reinfecções por até quatro semanas. No entanto, no Brasil, esta técnica, que oferece uma boa alternativa para controlar $H$. contortus por um período de até seis semanas (Soli et al 2010), não tem sido utilizada comercialmente.

Quarentena. A falta de quarentena em animais recém-introduzidos é uma falha no manejo, que pode acarretar na introdução de cepas resistentes na propriedade (Torres-Acosta \& Hoste 2008). Nos animais adquiridos deve ser realizado um tratamento utilizando mais de uma classe de ANTH e só devem ser introduzidos na pastagem após os exames de contagem de ovos nas fezes (OPG) serem negativos (Torres-Acosta \& Hoste 2008).

Fungos nematófagos. Fungos nematófagos são fungos com habilidade de invadir e matar as larvas dos nematóides nas fezes. A administração desses fungos por via oral tem demonstrado ser uma alternativa importante para reduzir a contaminação ambiental por larvas infectantes (L3). $\mathrm{O}$ principal fungo utilizado como controle biológico é $\mathrm{Du}$ ddingtonia flagrans. (Hoste \& Torres-Acosta 2011, Torres-Acosta et al. 2012). No semiárido paraibano experimentos com $D$. flagrans administrado a caprinos, em pellets, reduziram significativamente o número de $\mathrm{OPG}$ e o número de parasitas encontrados em caprinos traçadores e aumentaram o ganho de peso e os valores de hematócrito (Vilela et al. 2012). No entanto a utilização deste fungo depende de resolver problemas tecnológicos para a sua produção continuada a custos acessíveis e a sua distribuição permanente nas áreas onde serão utilizados.

Controle por OPG e tratamentos táticos. Recentemente foram acompanhados oito rebanhos de caprinos leiteiros no semiárido paraibano realizando exames coprológicos mensais e tratando o rebanho quando a contagem de OPG atingia 500-1000. Apesar da ocorrência de resistência múltipla, durante um período de 29 meses não ocorreram casos clínicos de parasitoses gastrintestinais e foram realizados em média $3,1 \pm 0.7$ tratamentos anuais. No primeiro ano, com precipitações dentro da normalidade para a região, foram realizados 3,8 tratamentos, sendo $2,3 \pm 1.1$ tratamentos na chuva e 1,5 \pm 0.8 na seca (Riet-Correa 2013). Esses resultados mostraram que mesmo com problemas de resistência é possível controlar as parasitoses gastrintestinais com três tratamentos durante a chuva e um durante 
a seca. Considerando as dificuldades dos produtores para realizarem exames de fezes sistemáticos, essa informação pode ser utilizada para determinar tratamentos táticos que não favoreçam o aumento da resistência. Para isso, o primeiro tratamento deveria ser realizado pelo menos 1530 dias após o início das chuvas após a contaminação das pastagens ou, alternativamente, quando o produtor observe algum sinal clínico de parasitose gastrintestinal. Posteriormente poderiam ser realizados um ou dois tratamentos durante a chuva, com intervalos de 30-40 dias. Por outro lado, é evidente que tratamentos na seca, pelo menos um, são também necessários, provavelmente por que nessa época ocorrem as maiores restrições na alimentação. Considerando que mesmo um único tratamento durante a seca é altamente favorável ao desenvolvimento de resistência o correto seria adotar nessa época algum tratamento seletivo como, por exemplo, tratar somente os animais jovens ou as cabras no periparto ou mesmo as com maior produção de leite. Outra alternativa para o controle durante a seca, seria realizar contagem de OPG de todo o rebanho e tratar somente os animais com contagens mais altas, com mais de 500-1000 OPG, por exemplo (Riet-Correa 2013).

\section{CONSIDERAÇÕES FINAIS}

Observa-se que tecnologias simples, que se aplicadas conjuntamente poderiam reduzir os prejuízos das parasitoses gastrintestinais, e inclusive retardar o crescimento da RA, ainda não são utilizadas nas regiões semiáridas do Brasil. É também preocupante o fato de que práticas que comprovadamente favorecem o surgimento da RA ainda serem utilizadas na região. Porém, apesar do controle das PGI ainda ser um dos maiores desafios para a caprinocultura da Região Nordeste, a utilização adequada do conhecimento já disponibilizado assim como daquele que vem sendo obtido a partir de estudos realizados sobre a epidemiologia destas enfermidades na região reduzirá a ocorrência e os prejuízos associados a estas.

\section{REFERÊNCIAS}

Amarante A.F.T., Bricarello P.A., Rocha R.A. \& Gennari S.M. 2004. Resistance of Santa Ines, Suffolk and Ile de France lambs to naturally acquired gastrointestinal nematode infections. Vet. Parasitol. 120:91-106.

Araujo Filho J.A. \& Carvalho F.C. 1998. Fenologia e valor nutritivo de espécies lenhosas caducifólias da caatinga. Comunicado Técnico Embrapa no 39, p.1-5.

Bang K.S., Familton A.S. \& Sykes A.R. 1990. Effect of copper oxide wire particle treatment on establishment of major gastro-intestinal nematodes in lambs. Res. Vet. Sci. 49:132-137.

Barger I.A., Siale K., Banks D.J.D. \& Le Jambre L.F. 1994. Rotational grazing for control of gastrointestinal nematodes of goats in a wet tropical environment. Vet. Parasitol. 53:109-116.

Barger I. 1997. Control by management. Vet. Parasitol. 72:493-506.

Barreto M.A. \& Silva J.S. 1999. Avaliação da resistência de nematódeos gastrintestinais em rebanhos caprinos do estado da Bahia (Resultados Preliminares). In: Seminário Brasileiro de Parasitologia Veterinária, Salvador. Colégio Brasileiro de Parasitologia Veterinária, p.160.

Burke J.M., Terrill T.H., Kallu R.R., Miller J.E. \& Mosjidis J. 2007. Use of copper oxide wire particles to control gastrointestinal nematodes in goats. J. Anim. Sci. 85:2753-276.

Burke J.M., Soli F., Miller J.E., Terrill T.H., Wildeus S., Shaik S.A., Getz W.R.
\& Vanguru M. 2010. Administration of copper oxide wire particles in a capsule or feed for gastrointestinal nematode control in goats Vet. Parasitol. 168(3/4):346-350.

Charles T.P., Pompeu J. \& Miranda D.B. 1989. Efficacy of three broad-spectrum anthelmintics against gastrointestinal nematode infections of goats. Vet. Parasitol. 34:71-75.

Chartier C. \& Hoste H. 1997. Response to challenge infection with Haemonchus contortus and Trichostrongylus colubriformis in dairy goats. Differences between high and low-producers. Vet. Parasitol. 73:267-276.

Chartier C., Pors I., Hubert J., Rocheteau D., Benoit C. \& Bernard N. 1998. Prevalence of anti-helmintic resistant nematodes in sheep and goats in Western France. Small Rumin. Res. 29(1):33-41.

Codevasf 2011. Manual de Criação de Caprinos e Ovinos. Brasília. 71p.

Coelho W.A.C. 2009. Resistência anti-helmíntica em caprinos no município de Mossoró/RN. Dissertação de Mestrado, Universidade Federal do Semiárido, Patos. 57p.

Costa V.M.M., Simões S.V.D. \& Riet-Correa F. 2009. Doenças parasitárias em ruminantes no semi-árido brasileiro. Pesq. Vet. Bras. 9(7):563-568.

Costa V.M.M., Simões S.V.D. \& Riet-Correa F. 2011. Controle das parasitoses gastrintestinais em ovinos e caprinos na região semiárida do Nordeste do Brasil. Pesq. Vet. Bras. 31(1):65-71.

Echevarria F.A.M. \& Trindade G.N.P. 1989. Anti-helmintic resistance by $\mathrm{Ha}$ emonchus contortus to ivermectin in Brazil. Vet. Rec. 124:147-148.

Embrapa. 1994. Recomendações tecnológicas para a produção de caprinos e ovinos no Estado do Ceará. Circ. Téc. № 9 , Embrapa-CNPC, Campo Grande, MS. 58p.

Gonçalves I.G. \& Echevarria F. 2004. Cobre no controle da verminose gastrintestinal em ovinos. Ciência Rural 34(1):183-188

Hart S. 2011. Effective and sustainable control of nematode parasites in small ruminants: The need to adopt alternatives to chemotherapy with emphasis on biologic control. 5o Simpósio Internacional Sobre Caprinos e Ovinos, João Pessoa, PB. (CD-ROM)

Hoste H., Frileux Y. \& Pommaret A. 2001. Distribution and repeatability of faecal egg counts and blood parameters in dairy goats naturally infected with gastrointestinal nematodes. Res. Vet. Sci. 70:57-60.

Hoste H., Chartier C., Lefrileux Y., Goudeau C., Broqua C., Pors I., Bergeaud J.P. \& Dorchies P.H.. 2002a. Targeted application of anthelmintics to control trichostrongylosis in dairy goats: result from a 2-year survey in farms. Vet. Parasitol. 110:101-108.

Hoste H., Le Frileux Y. \& Pommaret A. 2002b. Comparison of selective and systematic treatments to control nematode infection of the digestive tract in dairy goats. Vet. Parasitol. 106:345-355.

Hoste H., Frileux Y., Goudeau C., Charter C., Pors I., Broqua C. \& Bergeaud J.P. 2002c. Distribution and repeatability of nematode faecal egg counts in dairy goats: a farm survey and implications for worm control. Res. Vet. Sci. 72:211-215.

Hoste H., Torres Acosta J.F.J., Paolini V., Aguilar Caballero A.J., Etter E., Lefrileux Y., Chartier C. \& Broqua C. 2005. Interactions between nutrition and gastrointestinal infections with parasitic nematodes in goats. Small Rumin. Res. 60:14151

Hoste H., Rulie A.C., Prevot F., Bergeaud J.P., Grisez C., De La Farge F., Jacquiet P. \& Dorchies P. 2006. Differences in receptivity to gastrointestinal infections with nematodes in dairy ewes: Influence of age and of the level of milk production. Small Rum. Res. 63:150-155.

Hoste H., Sotiraki S., Landau S.Y., Jackson F. \& Beverige I. 2010. Goat- nematode interactions: think differently. Trends Parasitol. 26(8):376-381.

Hoste H. \& Torres-Acosta J.FJ. 2011. Non chemical control of helminths in ruminants: adapting solutions for changing worms in a changing world. Vet. Parasitol. 180:144-154.

Jackson F., Varady M. \& Bartley D.J. 2012. Managing anthelmintic resistance in goats: can we learn lesson from sheep? Small Rumin. Res. 103:3-9.

Lima W.C., Athayde A.C.R., Medeiros G.R., Lima A.S.D., Borburema J.B.B., Santos E.M., Vilela V.L.R. \& Azevedo S.A. 2010. Nematóides resistentes a alguns anti-helmínticos em rebanhos caprinos no Cariri Paraibano. Pesq. Vet. Bras. 30(12):1003-1009. 
Marie-Magdeleine M., Mahieu M., Philibert L., Despois P. \& Archimède H. 2010. Effect of cassava (Manihot esculenta) foliage on nutrition parasite infection and growth of lambs. Small Rumin. Res. 93:10-18.

Melo A.C.F.L., Reis I.F., Bevilaqua C.M.L.,Vieira L.S., Echevarria F.A.M. \& Melo L.M. 2003. Nematódeos resistentes a anti-helmínticos em rebanhos de ovinos e caprinos do Estado do Ceará, Brasil. Ciência Rural 33(2):339-344.

Monteiro J.M., Lins Neto E.M.F., Amorim E.L.C., Strattmann R.R., Araújo E.L. \& Albuquerque U.P. 2005. Teor de taninos em três espécies medicinais arbóreas simpátricas da caatinga. Revta Árvore 29(6):999-1005.

Molento M.B. 2008. Método FAMACHA: tratamento seletivo no controle de Haemonchus contortus, p.25-32. In: Veríssimo J.C. (Ed.), Alternativas de Controle da Verminose em Pequenos Ruminantes. Instituto de Zootecnia, Nova Odessa, SP.

Molento M.B., Fortes F.S., Pondelek D.A.S., Borges F.A., Chagas A.C.S., Torres-Acosta J.F. \& Geldhof P. 2011. Challenges of nematode control in ruminants: focus on Latin America. Vet. Parasitol. 180:126-132.

Muir J.P. 2011. The multi-faceted role of condensed tannins in the goat ecosystem. Small Rum. Res. 98:115-120.

O'Connor L.J., Brown-Walkden S.W. \& Kahn L.P. 2006. Ecology of the free-living stages of major trichostrongylid parasites of sheep. Vet. Parasitol. 142:1-15.

Pomroy W.E., Hart S.P. \& Min B.R. 2003. Rotational grazing as a parasite management tool for goats. J. Anim. Sci. 80(1):193.

Pomroy W.E. 2006. Anthelmintic resistance in New Zealand: a perspective on recent findings and options for the future. N. Z. Vet. J. 54:265-270.

Riet-Correa F. 2004. Suplementação mineral em pequenos ruminantes no semi-árido. Ciênc. Vet. Trópicos, Recife, 7(3/4):112-130.

Riet-Correa B. 2013. Assistência técnica integral à caprinocultura leiteira no semiárido com ênfase no controle parasitário. Tese de Doutorado, Universidade Federal da Paraíba. 64p.

Riet-Correa B., Simões S.V.D., Pereira Filho J.M., Azevedo S.S.A., Melo D.B., Batista J.A. \& Riet-Correa F. 2013. Sistemas produtivos de caprinocultura leiteira no semiárido paraibano: caracterização, principais limitantes e avaliação de estratégias de intervenção. Pesq. Vet. Bras. 33(3):345-352.
Rinaldi L. \& Cringoli G. 2012. Parasitological and pathophysiological methods for selective application of anthelmintic treatments in goats. Small Rum. Res. 103:18-22.

Santos V.T. \& Gonçalves P.C. 1967. Verificação de estirpes resistentes de Haemconchus contortus resistente ao thiabendazole no Rio Grande do Sul, Brasil. Revta Fac. Agron. Vet., Porto Alegre, 9:201-209.

Sebrae 2010. Manejo Básico de Ovinos e Caprinos. SEBRAE, Brasília. 137p. Soli F., Terrill T.H., Shaik S.A., Getz W.R., Miller J.E., Vanguru M. \& Burke J.M. 2010. Efficacy of copper oxide wire particles against gastrointestinal nematodes in sheep and goats. Vet. Parasitol. 168(1/2):93-96.

Torres-Acosta J.F.J. \& Hoste H. 2008. Alternative or improved methods to limit gastro-intestinal parasitism in grazing sheep and goats. Small Rumin. Res. 77:159-173.

Torres-Acosta J.F.J., Sandoval-Castro C.A., Hoste H., Aguilar Caballero, Cámara-Sarmiento R. \& Alonso-Diaz M.A. 2012. Nutritional manipulation of sheep and goats for the controlo $f$ gastrointestinal nematodes under hot humid and subhumid tropical conditions. Small Rum. Res. 103:2840.

Van Wyk J.A., Stenson M.O., Van der Merwe J.S., Vorster R.J. \& Viljoen P.G. 1999. Anthelmintic resistance in South Africa: surveys indicate an extremely serious situation in sheep and goat farming. J. Vet. Res. 66(4):273284.

Van Wyk J.A. \& Bath G.F. 2002. The FAMACHA system for managing haemonchosis in sheep and goats by clinically identifying individual animals for treatment. Vet. Res. 33:509-529.

Vieira L.S. \& Cavalcante A.C.R. 1999. Resistência anti-helmíntica em rebanhos caprinos no Estado do Ceará. Pesq. Vet. Bras. 19(3/4):99-103.

Villalba J.J., Provenza F.D., Hall J.O. \& Lisonbee L.D. 2010. Selection of tannins by sheep in response to gastrointestinal nematode infection. J. Anim. Sci. 88:2189-2198.

Vilela V.L.R., Feitosa T.F., Braga F.R., Araújo J.V., Souto D.V.O., Santos H.E.S., Silva G.L.L. \& Athayde A.C.R. 2012. Biological control of goat gastrointestinal helminthiasis by Duddingtonia flagrans in a semi-arid region of the northeastern Brazil. Vet. Parasitol. 188(1/2):127-133. 